\title{
Cutaneous Larva migrans: 3 cases at the forehead
}

\section{Laouali Salissou', Sareye Ousmane', Moussa Doulla', Souleymane Brah², Mamane Daou ${ }^{2}$, Djibo Ali ${ }^{3}$, Eric Adehossi}

${ }^{1}$ Department of Dermatology, National Hospital Niamey, Niamey, Niger, ${ }^{2}$ Internal Medicine, National Hospital Niamey, Niamey, Niger Service, ${ }^{3}$ Department of Infectious Diseases, National Hospital Niamey, Niamey, Niger

Corresponding author: Dr. Laouali Salissou, E-mail: danmata@yahoo.com

\begin{abstract}
Cutaneous Larva migrans, known as creeping dermatitis, is a parasitic disease caused by an hookworm larvae in a dead end in moist soil. It is a very common condition in tropical countries, especially among children playing in sand and among travelers. The body contact with soil contaminated with larvae in certain religious practices may expose individuals to contamination. We report three cases of CML at the forehead in three Muslim religious practitioners. Contamination was made by contact with dirty soil in unfenced mosques during overwintering. The presence of stray cats was confirmed by the 3 patients. Treatment with albendazole resulted in complete healing.
\end{abstract}

Key words: Cutanéous larva migrans; Forehead localisation; Albendazole; Niger

How to cite this article: Salissou L, Ousmane S, Doulla M, Brah S, Daou M, Ali D, Adehossi E. Cutaneous Larva migrans: 3 cases at the forehead. Our Dermatol Online. 2017;8(Suppl. 1):36-39.

Submission: 28.06.2017; Acceptance: 21.07.2017

DOI: 10.7241 /ourd.2017s.9 


\title{
Larva migrans cutanée: 3 cas de localisation au front $s$
}

\section{Laouali Salissou', Sareye Ousmane', Moussa Doulla', Souleymane Brah², Mamane Daou ${ }^{2}$, Djibo Ali ${ }^{3}$, Eric Adehossi ${ }^{2}$}

\author{
${ }^{1}$ Department of Dermatology, National Hospital Niamey, Niamey, Niger, ${ }^{2}$ Internal Medicine, National Hospital Niamey, \\ Niamey, Niger Service, ${ }^{3}$ Department of Infectious Diseases, National Hospital Niamey, Niamey, Niger
}

Corresponding author: Dr. Laouali Salissou, E-mail: danmata@yahoo.com

\begin{abstract}
RÉSUMÉ
La Larva Migrans Cutanée (LMC) connue sous le nom de larbisch ou creeping disease est une maladie parasitaire due à une larve d'ankylostome en impasse dans le sol humide. C'est une affection très fréquente dans les pays tropicaux notamment chez les enfants jouant dans le sable et chez les voyageurs. Le contact du corps avec le sol souillé de larves dans certaines pratiques religieuses, peut exposer les individus à la contamination. Nous rapportons 3 cas de LMC à localisation au front chez 3 pratiquants de religion musulmane. La contamination a été faite par contact avec le sol souillé par les excréments d'animaux dans des mosquées non clôturées pendant l'hivernage. La présence de chats errants a été confirmée par les 3 patients. Le traitement par l'albendazole a entrainé la guérison totale.
\end{abstract}

Mots clés: Larva migrans cutanée; Localisation frontale; Albendazole; Niger

\section{INTRODUCTION}

La larva Migrans Cutanée (LMC) est une parasitose cosmopolite, aussi bien rapportée dans les zones tropicales que dans les zones tempérées [1-3]. Elle est décrite la première fois en 1874 par Lee RJ [4], comme une affection cutanée bénigne, occasionnée par la migration accidentelle d'une larve de nématode d'animaux dans la partie superficielle de la peau. Dans les pays tropicaux et subtropicaux les larves les plus incriminées sont: Ancylostoma caninum, Ancylostoma brasiliense et Uncinaria stenocephala [5]. La présence d'animaux domestiques dans l'environnement, notamment le chien et le chat est un facteur aidant au diagnostic.

Nous rapportons 3 cas de LMC, observés pendant la saison hivernale, de localisation rare au niveau de front, survenus après le contact avec le sol humide lors de la prière. Le traitement par l'albendazole par voie orale a entrainé la guérison dans tous les cas, sans effets secondaires.

\section{OBSERVATION}

Il s'agissait de trois hommes dont l'âge variait de 30 à 60 ans et tous pratiquants de religion musulmane que nous avons reçus en consultation au service de Dermatologie de l'Hôpital National de Niamey au Niger. La durée de la maladie avant la consultation variait de 3 à 6 semaines. Linterrogatoire révélait surtout la présence dans l'environnement des patients, de chats errants dans leurs mosquées non clôturées. Un traitement ne précisant pas les médicaments utilisés a été effectué localement et par voie générale, par chacun et sans résultat. Le prurit avec des lésions excoriées ou non ont été le motif de la consultation. A l'examen on notait des lésions excoriées (Figs. 1 et $3 a$ et $3 b$ ) chez les 3 patients, ou des sillons serpigineux typiques dans 2 cas (Figs. 1 et 2). Ces lésions plus ou moins nombreuses étaient localisées au front, exactement au lieu de contact avec le sol lors de la prière chez le musulman. Les trois patients ont effectué pendant 3 jours consécutifs la prise d'albendazole, à raison $400 \mathrm{mg} /$ jour par voie orale. Des soins locaux par

\footnotetext{
How to cite this article: Salissou L, Ousmane S, Doulla M, Brah S, Daou M, Ali D, Adehossi E. Larva migrans cutanée: 3 cas de localisation au front. Our Dermatol Online. 2017;8(Suppl. 1):36-29.

Submission: 28.06.2017; Acceptance: 21.07.2017

DOI: 10.7241 /ourd.2017s.9
} 
attouchement des lésions avec la povidone iodée ont été effectués. La guérison a été obtenue après les trois prises d'albendazole sans effets secondaires avec un suivi de 8 semaines (Figs. $4 a$ et $4 b$ ).

\section{DISCUSSION}

La LMC est rapportée dans les pays tropicaux et également dans les pays tempérés [1-3]. La tranche d'âge de 1 à 5 ans est la plus atteinte [6]; cependant les adultes semblent plus atteints lors de voyage du fait de la présence des chats rôdant sur les plages et les hôtels [7-9], et de l'humidité facteur déterminant à la survie des larves. Le contact du front avec le sol mouillé pendant la période hivernale, était déterminant. Tous les trois cas étaient survenus pendant la période hivernale au Niger: Le premier cas en octobre 2010, le deuxième en Août 2013 et le troisième en Août 2016. Chez nos patients comme c'est le cas habituel, le

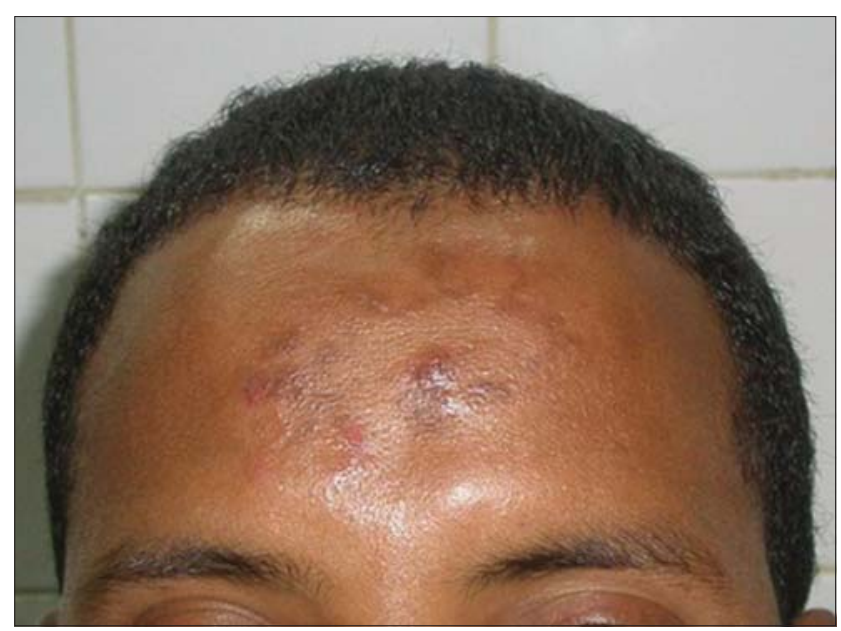

Figure 1: Lésions avec excoriation.

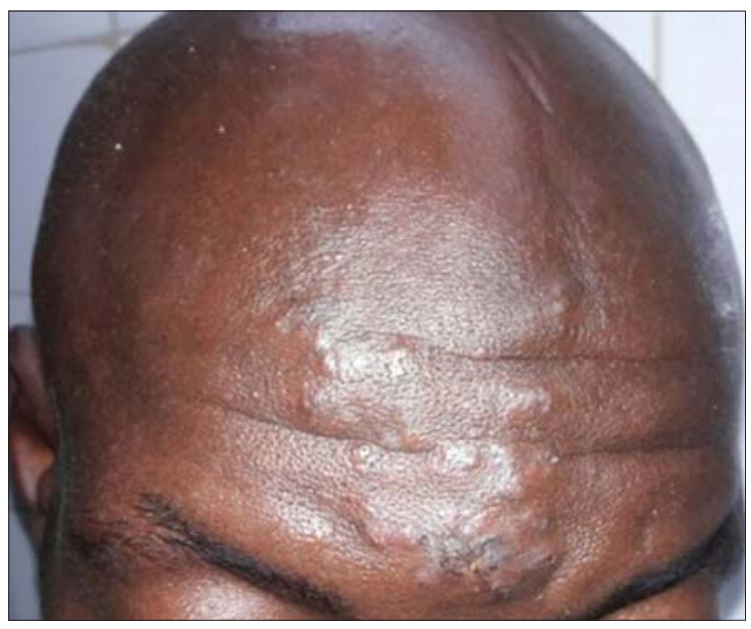

Figure 2: Multiples lésions serpigineuses. prurit était le principal motif de consultation $[3,9,10]$. La lésion serpigineuse était objectivement présente dans 2 cas, mais est toujours la plus observée dans les consultations $[3,7,8,10]$. La localisation des lésions se fait surtout sur les zones le plus en contact avec le sol souillé et humide et concerne les membres pelviens $[7,11,12]$; cette localisation concerne également d'autres régions inhabituelles $[13,14]$. La particularité de nos cas, est aussi leur localisation au front, favorisée par le contact avec le sol souillé et humide lors de la prière chez les trois patients pratiquants musulmans.

De nombreuses molécules utilisées seules ou en association ont variablement prouvé leur efficacité dont les principales sont: l'albendazole, le thiabendazole, le mébendazole, le fluvermal, et/ ou l'ivermectine [15-18]. Dans nos trois cas, la prise d'albendazole en 3 jours consécutifs, a entrainé la guérison sans effets secondaires avec un suivi de 8 semaines.

\section{CONCLUSION}

La larva migrans est classiquement favorisée par l'humidité et les animaux hôtes domestiques principalement le chat et le chien. La localisation au front est rare et peut être favorisée par la pratique de certaine religion. Le traitement à base d'albendazole est toujours efficace.

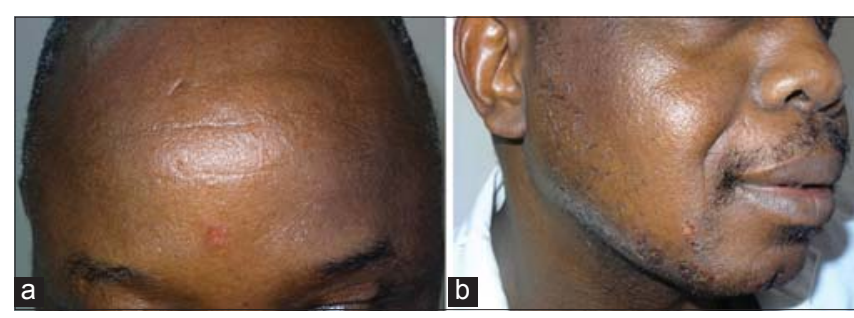

Figure 3: (a) Lésion excoriée avant traitemet. (b) Lesions excoriées avant traitement.

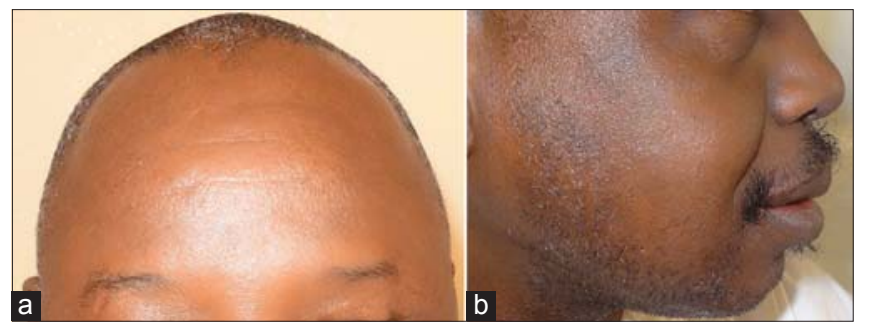

Figure 4: $(a$ et $b)$ Disparition des lésions après traitement. 


\section{www.odermatol.com}

\section{REFERENCES}

1. Tamminga N, Bierman Wouter FW, De Vries PJ. Cutaneous Larva Migrans acquired in Brittany France. Emerg infect Dis. 2009;15:1856-7.

2. Antonio K, Ralf B, Peter H. Hoeger. Cutaneous larva Migrans in northern Germany. Eur J Pediatr. 2007;166:1183-5.

3. Camara A, Camara AD, Baldé H, Soumah MM, Keita M, Doumbouya A, et al. Larva migrans cutanée: aspect épidémiologique, clinique et thérapeutique. Ann Dermatol Vénérol. 2011;138s:296.

4. Lee RJ. Case of creeping eruption. Trans clin Soc London. 1874;8:44-5.

5. Romain B, Christian C, Tristan F. Imported cutaneous larva migrans by a 31- year-old French woman after a travel in Gabon. BMJ Case Rep. 2016;12;2016. pii: bcr2016216578.

6. Salissou L, Adehossi E, Brah S, Gado M, MaguiaT, Kanga J.M. Larva Migrans Cutanée: Aspect épidémiologique, Clinique, et thérapeutique à propos de 73 cas au Centre National Dermatolèpre de Niamey (Niger). Ann Univers ABDOU M, Tome XIII-A, PP72-76, 2012.

7. Jelineck T, Maïwald H, Northdurft HD, Löscher T. Cutaneous Larva Migrans in travelers: synopsis of histories, symptoms and treatements of 98 patients. Clin Infect Dis. 1994;19:1062-6.

8. Caumes E, Carrière J, Guermonprez G, Bricaire F, Danis M, Gentilini M. Dermatosis Associated with Travel to Tropical Countries: A prospective study of the diagnosis and management of 269 patients presenting to a tropical disease Unit. Clin Infect Dis. 1995;20:542-8.

9. Bouchaud O, Houzé S, Schiemann R, Durand R, Ralaimazaba P, Ruggeri $\mathrm{C}$, et al. Cutaneous larva migrans in travelers: A prospective study, with assesment of therapy with ivermectin. Clinl Infect Dis. 2000;31:493-98.
10. Davies HD, Sakuls P, Keystone JS. Creeping Eruption. A review of clinical presentation and management of 60 cases presenting to a tropical disease Unit. Arch Dermatol. 1993;129:588-91.

11. Prudhomme L, Loche F, Massip P, Marchou B. Larva migrans cutanée: Echec de l'ivermectine en dose unique. Méd Mal Infect. 2002;32:115-18.

12. Torres J, Orihuela A, Garcia D, Abdul-Hadi S. Treatment of cutaneous larva migrans with Albendazole. Preliminary Report. Rev Inst Med trop Sao Paulo. 1989;31:56-8.

13. Meotti PCD, Plates G, Nogueira LLC, Silva RA, Paolini KS, Nunes EM, et al., Cutaneous larva migrans on the scalp: unusual presentation in a typical clinical presentation. An Bras Dermatol. 2014;89:332-3.

14. Sugathan P, Bhagyanathan M. Cutaneous Larva Migrans: Presentation at an Unusual Site. Indian J Dermatol. 2016;61:574-5.

15. Tremblay A, Maclean JD, Gyorkos T, MacPherson DW. Outbreak of cutaneous larvamigrans in a group of travellers. Trop Med Int Health. 2000;5:330-34.

16. Padmavthy L, Rao LL. Cutaneous larva migrans: A case report. Indian J Med Microbiol. 2005;23:135-6.

17. Chiriac A, Birsan C, Chiriac AE, Murgu A, Solovan C. Cutaneous larva migrans: report of three cases with response to Albendazole. Our Dermatol Online. 2012;3:126-7.

18. Paul IS, Singh B. Cutaneous larva migrans in children: A case series from southern India. Indian J Paediatr Dermatol. 2017;18:36-8.

Copyright by Laouali Salissou, et al. This is an open-access article distributed under the terms of the Creative Commons Attribution License, which permits unrestricted use, distribution, and reproduction in any medium, provided the original author and source are credited.

Source of Support: Nil, Conflict of Interest: None declared. 